\title{
Percutaneous mechanical thrombectomy in the treatment of acute iliofemoral deep vein thrombosis: a systematic review
}

\author{
PC Wong, YC Chan *, Y Law, Stephen WK Cheng
}

\section{A B S T R A C T}

Background: Conventional treatment of deep vein thrombosis (DVT) of the lower extremities by anticoagulation alone has been proven to be insufficient to prevent recurrence and postthrombotic syndrome (PTS). Early restoration of venous patency and preservation of valvular function by endovascular surgery has been advocated. The aim of this study was to review the efficacy and safety of percutaneous mechanical thrombectomy (PMT) against catheter-directed thrombolysis (CDT) in the treatment of acute iliofemoral DVT.

Methods: Three hundred sixty-nine articles were identified through screening of the PubMed, EMBASE, and Cochrane databases from January 2006 to December 2016.

Results: Fifteen retrospective studies and one prospective registry, totalling 1170 patients, were recruited for qualitative synthesis. The venous patency rate ranged from $75 \%$ to $100 \%$ with mean follow-up of 12.3 months. The rates of PTS and

This article was published on 14 Jan 2019 at www.hkmj.org. recurrent DVT were less than $17 \%$ and $15 \%$, respectively. The overall mortality rate was $0.26 \%$.
Compared with CDT, PMT was shown to reduce PTS at 1 year (Villalta score: $2.1 \pm 3.0$ in the PMT group and $5.1 \pm 4.1$ in the CDT group, $\mathrm{P}=0.03$ ) and bleeding complications (packed cells transfused: $0.2 \pm$ 0.3 units in the pharmacomechanical thrombectomy group and $1.2 \pm 0.7$ units in the CDT group, $\mathrm{P}<0.05$ ).

Conclusion: Percutaneous mechanical thrombectomy is a safe and effective treatment for acute iliofemoral DVT in terms of restoration of venous patency, prevention of DVT recurrence, and PTS. Compared with CDT alone, PMT offers a lower risk of PTS and bleeding complications.

\section{Hong Kong Med J 2019;25:48-57 \\ https://doi.org/10.12809/hkmj18749}

PC Wong, MB, BS

YC Chan *, MB, BS, MD

Y Law, MB, BS

SWK Cheng, MB, BS, MS

Department of Surgery, Queen Mary Hospital, Pokfulam, Hong Kong

* Corresponding author: ycchan88@hkucc.hku.hk

\section{Introduction}

Deep vein thrombosis (DVT) is a major cause of morbidity and mortality, as it can lead to postthrombotic syndrome (PTS) and pulmonary embolism. According to the American College of Chest Physicians treatment guidelines, DVT has conventionally been treated with low-molecularweight heparin, unfractionated heparin, or fondaparinux followed by vitamin $\mathrm{K}$ antagonists for at least 3 months. ${ }^{1}$ This recommended regimen is adequate for prevention of thrombus extension, but its effect on clot lysis is minimal. The reported 6-month venous patency rate in patients treated with anticoagulation alone was only $47.4 \%$. Eventually, up to $55.6 \%$ of patients with iliofemoral DVT developed PTS as a result of valvular dysfunction. ${ }^{2} \mathrm{Up}$ to $5 \%$ to $10 \%$ of patients had severe PTS in the form of venous ulceration, which caused significant morbidity and socio-economic cost. ${ }^{3}$

In view of the suboptimal treatment outcomes of anticoagulation, aggressive means have been developed to achieve early restoration of venous patency and thus preservation of valvular function. A Cochrane review suggested that early thrombus removal by means of systemic thrombolysis can prevent venous dysfunction and PTS. However, its use was limited by its significantly increased risk of bleeding. ${ }^{4}$

Endovascular modalities including catheterdirected thrombolysis (CDT) and percutaneous mechanical thrombectomy (PMT) were developed to achieve accelerated thrombolysis with less bleeding risk. Catheter-directed thrombolysis was shown to be superior to anticoagulation alone in terms of higher thrombolysis rate and lower rates of recurrence and $\mathrm{PTS}^{2}{ }^{2}$ It features loco-regional delivery of thrombolytic agent over the DVT site via a transluminal catheter. The dosage of the thrombolytic agent can be reduced compared with that of systemic thrombolysis, and thus, a reduction in bleeding complications can be achieved. Its benefits have been validated by a number of randomised controlled trials and meta-analyses, but its application rate remains low because of its substantial bleeding risk and cost. ${ }^{5}$ 
Percutaneous mechanical thrombectomy is another form of endovascular treatment, in which thrombectomy devices are passed to the site of DVT and blood clots are removed by different mechanical means. It can also be used as an adjunctive device to CDT or pharmacomechanical thrombectomy. When these two devices are used in combination, the dosage of thrombolytic agents can be lowered further and the duration of procedure can be shortened. ${ }^{6}$ According to the American College of Chest Physicians guidelines, PMT provides the greatest benefits for young and functionally active patients with acute presentation $(<14$ days, or presence of phlegmasia cerulea dolens) of extensive proximal DVT. ${ }^{1}$ Percutaneous mechanical thrombectomy has provided promising results in various studies, while high-level evidence to guide its implementation is still lacking. Against this background, this article aimed to review the evidence about PMT regarding its procedural outcomes and safety profile in the treatment of DVT.

\section{Methods}

\section{Literature search}

A systematic review was conducted in accordance with the PRISMA (Preferred Reporting Items for Systematic reviews and Meta-Analysis) statement (http://www.prisma-statement.org/). An electronic search was performed using the PubMed, EMBASE, and Cochrane Databases from January 2006 to December 2016. The medical subject heading (MeSH) terms used were "mechanical thrombectomy" and "venous thrombosis" or "deep vein thrombosis".

\section{Study selection}

The inclusion criteria were as follows: DVT of the lower extremities; human study; study population aged $\geq 18$ years; and articles published in English. Reviews and case reports were excluded. All studies of interest were obtained as full-text articles and assessed by two authors independently. Final decisions on inclusion in the study were made by the entire research team.

\section{Data extraction and outcome measurement}

Relevant data were extracted with the following items recorded: author, title, year of publication, number and age of patients, co-morbidities, duration of follow-up, onset of symptoms, location of DVT, type of thrombectomy device, and adjunctive modalities. Efficacy was measured in terms of rates of venous patency, recurrence, and PTS. Complications including bleeding, pulmonary embolism, and mortality were recorded. Secondary outcomes included dosage of thrombolytic agents, cost, and duration of procedure.

\section{經皮機械血栓切除術治療急性髂股深靜脈血栓： 系統綜述 \\ 黃柏釗、陳燿志、羅旭、鄭永強}

背景：單獨使用抗凝治療下肢深靜脈血栓（DVT）已被證實不足以預 防復發和血栓後綜合徵（PTS）, 故提倡以血管內手術及早恢復靜脈 通暢和保持瓣膜功能。本研究旨在回顧經皮機械血栓切除術（PMT） 對比導管溶栓 (CDT) 於治療急性髂股深靜脈血栓的療效和安全性。

方法：2006年1月至 2016 年 12 月期間於PubMed、EMBASE和 Cochrane數據庫節選369篇文獻。

結果：選定15項回溯性研究和一項前瞻性登記研究項目, 合共 1170 名患者進行定量研究分析。靜脈通暢率界乎 $75 \%$ 至 $100 \%$, 平均隨訪 期為 12.3 個月。血栓後綜合徵和深靜脈血栓復發率分別低於 $17 \%$ 和 $15 \%$ 。整體死亡率為 $0.26 \%$ 。與導管溶栓相比, 經皮機械血栓切除 術後 1 年內較少出現血栓後綜合徵（Villalta量表評分：5.1 14.1 比 $2.1 \pm 3.0, P=0.03$ ) 和出血併發症 (輸血需求 : 藥物機械血栓切除術 $0.2 \pm 0.3$ 單位比導管溶栓 $1.2 \pm 0.7$ 單位,$P<0.05)$ 。

結論：經皮機械血栓切除術治療急性髂股深靜脈血栓是安全有效的治 療, 可恢復靜脈通暢、預防深靜脈血栓復發和血栓後綜合徵。與單獨 使用導管溶栓相比，經皮機械血栓切除術可降低血栓後綜合徵和出血 併發症的風險。

\section{Data analysis}

Statistical meta-analysis was not performed because of the heterogeneity of the original data. Therefore, descriptive data were summarised and presented in tables to provide a comprehensive overview of different clinical aspects of the studies.

\section{Results}

Our initial search yielded a total of 369 articles, including 260 articles from PubMed, 98 articles from EMBASE, and 11 studies from the Cochrane Library. Thirty-one duplicated records and 283 irrelevant studies were excluded upon screening of titles and abstracts, leaving 55 potentially eligible studies. A further 39 articles were excluded after full-text articles were assessed: 28 review articles; one study on the patients with inferior vena cava (IVC) filter; one study on the effect of clot age; seven studies without full text; and two non-English studies. Fifteen retrospective studies and one prospective registry were included into our analysis, in which seven articles reported comparative evidence of PMT versus CDT. There were no published randomised trials available.

Baseline patient demographics and characteristics of the studies are summarised in Table $1 .^{6-21} \mathrm{~A}$ total of 1170 patients were included (range, 16-329 patients) with a mean age of 53.5 years (range, 16 to 88 years). The mean follow-up time was 12.3 months (range, 1-82 months). 
TABLE I. Patient demographics and study characteristics ${ }^{6-21}$

\begin{tabular}{|c|c|c|c|c|c|}
\hline First author, year & No. of patients & Age (years)* & $\begin{array}{l}\text { Follow-up period } \\
(\mathrm{mo})^{*}\end{array}$ & $\begin{array}{l}\text { Time since onset } \\
\text { (days) }^{*}\end{array}$ & Location of DVT \\
\hline Garcia, $2015^{6}$ & $\begin{array}{l}\mathrm{RT}=13 \\
\mathrm{PCDT}=115 \\
\mathrm{PCDT}+\mathrm{CDT}=172 \\
\mathrm{RT}+\mathrm{CDT}=29 \\
(\mathrm{n}=329)\end{array}$ & $52.2 \pm 16$ & 12 & $\begin{array}{l}\leq 14,67 \% \\
15-30,19 \% \\
>30,14 \%\end{array}$ & $\begin{array}{l}\text { Iliac, } 66 \% \\
\text { Femoral, } 89 \% \\
\text { Popliteal, 59\% }\end{array}$ \\
\hline Huang, $2015^{7}$ & $\begin{array}{l}\mathrm{PMT}=16 \\
\mathrm{CDT}=18 \\
(\mathrm{n}=34)\end{array}$ & $\begin{array}{l}\mathrm{PMT}=62.7 \pm 10.1 \\
\mathrm{CDT}=62.1 \pm 15\end{array}$ & 12 & - & - \\
\hline Arko, $2007^{8}$ & 30 & $50.9 \pm 18$ & $6.2(3-24)$ & $5.7(3-14)$ & $\begin{array}{l}\text { Iliofemoral = } 14 \\
\text { Iliofemoropopliteal = } 6 \\
\text { Femoropopliteal = } 5 \\
\text { Subclavian = } 5\end{array}$ \\
\hline Lin, $2006^{9}$ & $\begin{array}{l}\mathrm{PMT}=52 \\
\mathrm{CDT}=46\end{array}$ & $\begin{array}{l}\mathrm{PMT}=45 \pm 12 \\
\mathrm{CDT}=49 \pm 10\end{array}$ & $13(1-49)$ & $\begin{array}{l}\mathrm{PMT}=15(0-34) \\
\mathrm{CDT}=13(0-31)\end{array}$ & - \\
\hline Kim, $2006^{10}$ & $\begin{array}{l}\mathrm{PMT}+\mathrm{CDT}=19 \\
\mathrm{CDT}=26\end{array}$ & $\begin{array}{l}\mathrm{PMT}+\mathrm{CDT}=53 \pm 20.7 \\
\mathrm{CDT}=42.9 \pm 13.2\end{array}$ & $32.1 \pm 38.7$ & $\leq 14$ & - \\
\hline Kim, $2006^{11}$ & $\begin{array}{l}\mathrm{PMT}+\mathrm{CDT}=27 \\
\mathrm{CDT}=40\end{array}$ & $\begin{array}{l}\mathrm{PMT}+\mathrm{CDT}=43.1 \pm 13.8 \\
\mathrm{CDT}=45 \pm 16.3\end{array}$ & - & - & - \\
\hline Ozpak, $2016^{12}$ & 21 & $48.7 \pm 10.7(27-69)$ & 6 & $\leq 14$ & $\begin{array}{l}\text { Iliofemoral, } 71.4 \% \\
\text { Popliteal, } 28.6 \% \\
\text { Femoral, } 38.1 \%\end{array}$ \\
\hline Jia, $2016^{13}$ & 68 & $61.7 \pm 7.8$ & $21.9 \pm 6.3$ & $\leq 14$ & $\begin{array}{l}\text { Popliteal-iliofemoral }=29 \\
\text { Iliofemoral }=31 \\
\text { lliac }=8\end{array}$ \\
\hline Gagne, $2015^{14}$ & 139 (151 Limbs) & $59.7(16-88)$ & 12 & $8.5 \pm 10.8$ & $\begin{array}{l}\text { Iliofemoral, } 45 \% \\
\text { Iliofemoropopliteal, } 29.8 \% \\
\text { Femoral, } 2.7 \% \\
\text { Femoropopliteal, } 7.9 \% \\
\text { Iliac, 6.6\% } \\
\text { IVC/iliac, } 6.6 \%\end{array}$ \\
\hline Lee, $2013^{15}$ & $\begin{array}{l}A T+C D T=71 \\
C D T=29 \\
(n=100)\end{array}$ & $60.5 \pm 14.6$ & $12(1-82)$ & - & - \\
\hline Karahan, $2016^{16}$ & 67 & $42.5 \pm 21.3$ & - & - & Iliofemoral \\
\hline Bozkurt, $2015^{17}$ & 16 & $58.5(21-79)$ & 6 & $\leq 14$ & $\begin{array}{l}\text { Femoropopliteal = } 5 \\
\text { Iliofemoral = } \\
\text { Iliac }=1 \\
\text { IVC }=1\end{array}$ \\
\hline Park, $2014^{18}$ & $\begin{array}{l}\mathrm{MT}=23 \\
\mathrm{MT}+\mathrm{CDT}=30 \\
\mathrm{CDT}=45 \\
(\mathrm{n}=90,98 \text { limbs })\end{array}$ & $58 \pm 16$ & $16(0-78)$ & $7(3-13)$ & $\begin{array}{l}\text { Iliac: } \\
\text { CDT, 80\% } \\
\text { MT+CDT, 83\% } \\
\text { MT, 83\% } \\
\text { Femoral: } \\
\text { CDT, 96\% } \\
\text { MT+CDT, 97\% } \\
\text { MT, 96\% } \\
\text { Popliteal: } \\
\text { CDT, 69\% } \\
\text { MT+CDT, 80\% } \\
\text { MT, 78\% } \\
\text { Calf: } \\
\text { CDT, 56\% } \\
\text { MT+CDT, 67\% } \\
\text { MT, 63\% }\end{array}$ \\
\hline Shi, $2011^{19}$ & 25 & $54.2 \pm 15.7$ & $12.3 \pm 2.5$ & $6.3 \pm 2.5$ & $\begin{array}{l}\text { IVC }=5 \\
\text { lliofemoral = } 16 \\
\text { Femoropopliteal = } 9\end{array}$ \\
\hline Shi, $2009^{20}$ & 16 & $53.3 \pm 15.6$ & $13(6-35)$ & $4.9(1-15)$ & $\begin{array}{l}\text { Iliofemoropopliteal }=14 \\
\text { Iliofemoropopliteal+IVC = } 2\end{array}$ \\
\hline Lee, $2006^{21}$ & 25 & $56(28-64)$ & $16(12-33)$ & $7(3-13)$ & Extensive iliofemoral \\
\hline
\end{tabular}

Abbreviations: AT = aspiration thrombectomy; CDT = catheter-directed thrombolysis; DVT = deep vein thrombosis; IVC = inferior vena cava; $M T$ T = mechanical thrombectomy; MTS = May-Thurner syndrome; PCDT = pharmacomechanical catheter-directed thrombolysis; PMT = percutaneous mechanical thrombectomy; PTA = percutaneous transluminal angioplasty; RT = rheolytic thrombectomy; rtPA = recombinant tissue plasminogen activator; $\mathrm{SD}=$ standard deviation

* Data are shown as mean \pm SD and/or range, unless otherwise specified 


\begin{tabular}{|c|c|c|c|}
\hline Type of device & Thrombolysis & IVC filter & Angioplasty \pm stenting (indication) \\
\hline AngioJet & CDT, $61 \%$ & - & $\begin{array}{l}\text { PTA, 74\% } \\
\text { Stenting, 35\% }\end{array}$ \\
\hline AngioJet & Urokinase & - & $\begin{array}{l}\text { PTA (stenosis }<70 \% \text { ); wall stent } \\
\text { (stenosis recoiled) }\end{array}$ \\
\hline $\begin{array}{l}\text { Trellis }=18 \\
\text { AngioJet }=12\end{array}$ & $\begin{array}{l}\text { Tenecteplase for partial thrombus } \\
\text { removal }\end{array}$ & Temporary, 21/25 & $\begin{array}{l}\text { PTA+stenting }=17 \\
\text { PTA }=11\end{array}$ \\
\hline AngioJet & Urokinase, reteplase, rtPA & $\begin{array}{l}\text { Temporary }=28 \\
\text { Permanent }=15\end{array}$ & $\begin{array}{l}\text { PMT, } 82 \% \\
\text { CDT, } 78 \%\end{array}$ \\
\hline AngioJet & Urokinase & - & $\begin{array}{l}\text { PMT+CDT, } 15.8 \% \\
\text { CDT, } 23.1 \%\end{array}$ \\
\hline AngioJet & Urokinase & - & $\begin{array}{l}\mathrm{PMT}+\mathrm{CDT}, 15 \% \\
\mathrm{CDT}, 18 \%\end{array}$ \\
\hline Aspirex & - & All & $\begin{array}{l}1 \text { PTA (femoral vein stenosis) } \\
2 \text { PTA+stenting } \\
\text { (iliac vein stenosis, MTS) }\end{array}$ \\
\hline Aspiration & $21 / 68$ & All & $\begin{array}{l}\text { Luminexx nitinol stent, } 47 \% \\
\text { (MTS/malignancy) }\end{array}$ \\
\hline Trellis & $\begin{array}{l}\text { CDT, } 30.1 \% \\
\text { AngioJet, } 5.3 \% \\
\text { EKOS, } 6.8 \%\end{array}$ & $\begin{array}{l}\text { Temporary }=29 \\
\text { Permanent }=57\end{array}$ & $\begin{array}{l}\text { PTA, } 74 \% \\
\text { Stenting, } 54 \%\end{array}$ \\
\hline Aspiration & Urokinase & $46 \%$ & $32 \%$ (MTS/>50\% residual thrombus) \\
\hline Cleaner & Reteplase & - & - \\
\hline Cleaner & Alteplase & $\begin{array}{l}\text { All except } 1 \text { with IVC chronic } \\
\text { obstruction }\end{array}$ & $\begin{array}{l}\text { PTA }=12 \\
\text { Wall stent }=9\end{array}$ \\
\hline Trerotola & Urokinase & $\begin{array}{l}\text { Temporary }=68 \\
\text { Permanent }=25\end{array}$ & Wall stent, 65\% (MTS) \\
\hline Amplatz & - & All & $\begin{array}{l}\text { PTA, 13/25 } \\
\text { Stenting, 11/25 (MTS) }\end{array}$ \\
\hline $\begin{array}{l}\text { Amplatz }=10 \\
\text { Rotarex }=6\end{array}$ & Urokinase & All & $\begin{array}{l}\text { PTA = } 3 \\
\text { Stenting = } 1 \text { (MTS) }\end{array}$ \\
\hline Trerotola, Aspiration & Urokinase & 5/25 (Contra-indicated to CDT) & $80 \%$ \\
\hline
\end{tabular}


Four different categories of thrombectomy devices were used among the included studies: rheolytic devices, ${ }^{6-11}$ aspiration devices, ${ }^{12-16}$ rotational devices, ${ }^{16-21}$ and ultrasound-enhanced thrombolysis devices. ${ }^{14}$ They aimed to achieve transcatheter removal of thrombi via different mechanical means.

The AngioJet system (Possis Medical, Minneapolis [MN], US) is a rheolytic device that generates high-velocity saline jets at the side of catheter, which create a localised low-pressure zone and thus result in maceration and aspiration of the thrombus.

During aspiration thrombectomy, the thrombus was aspirated out through the percutaneous catheter as the catheter was gradually pulled out. The process was repeated until complete removal of the thrombus or at least $90 \%$ disappearance of thrombi. Two of the aspiration systems were Aspirex (Straub Medical, Wangs, Switzerland) and the Trellis infusion system (Covidien, Mansfield [MA], US), which is a sophisticated system that contains an oscillation drive unit that mixes the thrombus with thrombolytic agents between two occlusion balloons.

Rotational devices feature high-frequency revolution of a helix that is controlled by a foot pedal. At least four different types of rotational devices were included, including the Amplatz thrombectomy device (Microvena, White Bear Lake [MN], US), Rotarex (Straub Medical, Wangs, Switzerland), Trerotola (Arrow International, Redding [PA], US), and Cleaner (Rex Medical, Fort Worth [TX], US and Argon Medical Devices, Inc, Plano [TX], US). They all consisted of a motor-driven fragmentation helix or basket that was rotated in the thrombosed vein. The thrombus was then aspirated out via the catheter.

An ultrasound-enhanced thrombolysis device (EKOS Corporation, Bothell [WA], US) was selectively used in one study ${ }^{14}$ for patients with inadequate thrombus removal despite the use of a rotational device. A high-frequency ultrasound wave was emitted through transducers inside the catheter to achieve maceration of the thrombus and mix it with thrombolytic agents.

\section{Efficacy}

Nine non-comparative and seven comparative studies were included in our analysis. Efficacy in terms of rates of venous patency, PTS, and recurrent thrombosis is shown in Table 2. ${ }^{6-21}$ Venous patency was measured most frequently by Duplex ultrasound $(n=9)$ followed by computed tomographic (CT) venography $(n=4)$ and contrast venography $(n=2)$. Imaging modalities were not mentioned in three studies. Venous patency was further quantified according to a 3-tier system in five studies: Grade I (<50\% clot lysis), Grade II (50\%-99\% clot lysis), and
Grade III (100\% clot lysis). ${ }^{22}$ Venous patency was measured at 6 months in four studies and at 1 year in the other 12 studies. Venous patency rates ranged from $75 \%$ to $100 \%$.

Rates of PTS were reported in terms of Villalta score $(n=2)^{23}$ or Venous Clinical Severity Score (VCSS) $[n=2] .{ }^{14}$ Four studies reported the rates of valvular incompetence from $8 \%$ to $24 \%$. The rates of DVT recurrence, reported in eight studies, ranged from $0 \%$ to $17 \%$.

\section{Complications}

The major complications of thrombectomy are shown in Table $3 .^{6-21}$ Six studies reported rates of pulmonary embolism ranging from $0.3 \%$ to $17 \%$, but none of them was clinically significant. No patient had pulmonary embolism in the remaining 11 studies. Garcia et $\mathrm{al}^{6}$ reported major bleeding complications in $3.6 \%$ of patients, including intracranial bleeding, gastrointestinal bleeding secondary to gastritis or gastric cancer, retroperitoneal bleeding, and haemolytic anaemia requiring transfusion. Minor bleeding complications were reported at frequencies of up to $28 \%$, most of which were access site bleeding. Blood transfusion and surgical intervention were seldom required. No operative mortality was reported in 12 studies, while only two cases of fatal intracranial haemorrhage were noted in two separate studies. Another mortality was reported by Garcia et $a l,{ }^{6}$ with an unknown cause of death. The overall mortality in this series was $0.26 \%$.

\section{Secondary outcomes}

Seven studies reported comparative evidence about PMT versus CDT (Table $4^{6,9,10,11,15,18}$ ). Huang et $\mathrm{al}^{7}$ showed that PMT significantly reduced PTS at 1 year, with lower Villalta scores in the PMT group $(2.1 \pm 3.0)$ than in the CDT group $(5.1 \pm 4.1$; Wilcoxon rank-sum test, $\mathrm{P}=0.03)$. However, no statistical difference was shown in Villalta scores in another retrospective study conducted by Park et al. ${ }^{18}$

Lin et $\mathrm{al}^{9}$ compared bleeding complications between the two groups in terms of the number of units of packed red blood cells transfused. There was a significant reduction of blood transfusion from $1.2 \pm 0.7$ units in the CDT group to $0.2 \pm 0.3$ units in the PMT group (Pearson Chi squared, $\mathrm{P} \leq 0.05){ }^{9}$

The dosage of thrombolytic infusion and average procedural time were significantly reduced in the CDT with adjunctive PMT group compared with the CDT alone group, as reported in at least four different retrospective studies..$^{10,11,15,18}$

From the economic perspective, two retrospective studies performed cost analysis, and PMT was found to be associated with $44 \%$ to $49 \%$ reduction in total hospital costs. ${ }^{9,10}$ It was also consistent with shorter hospital and intensive care unit (ICU) stays in the PMT group (4.6 \pm 1.3 days 
TABLE 2. Efficacy of percutaneous mechanical thrombectomy $y^{6-21}$

\begin{tabular}{|c|c|c|c|}
\hline First author, year & Patency rate & Post-thrombotic syndrome & DVT recurrence \\
\hline Garcia, $2015^{6}$ & $\begin{array}{l}1 \text { Year by venography } \\
\text { Grade III: } \\
\text { RT, 69.2\% } \\
\text { PCDT, 58.3\% } \\
\text { PCDT+CDT, 62.1\% } \\
\text { RT+CDT, 57.3\% } \\
\text { Grade II: } \\
\text { RT, 30.8\% } \\
\text { PCDT, 36.5\% } \\
\text { PCDT+CDT, 31\% } \\
\text { RT+CDT, 69.8\% } \\
\text { Grade I: } \\
\text { RT, 0\% } \\
\text { PCDT, 5.2\% } \\
\text { PCDT+CDT, 6.9\% } \\
\text { RT+CDT, 2.9\% }\end{array}$ & NR & $17 \%$ \\
\hline Huang, $2015^{7}$ & $\begin{array}{l}1 \text { Year by Duplex } \\
\text { PMT, } 93.8 \% \\
\text { CDT, } 88.9 \%\end{array}$ & $\begin{array}{l}\text { Villalta scores }(P=0.03) \\
\text { PMT, } 2.06 \pm 2.95 \\
\text { CDT, } 5.06 \pm 4.07\end{array}$ & NR \\
\hline Arko, $2007^{8}$ & 6.2 Months by Duplex, $90 \%$ & Valvular incompetence, $12 \%$ & NR \\
\hline Lin, $2006^{9}$ & $\begin{array}{l}1 \text { Year by venography } \\
\text { PMT, } 68 \% \\
\text { CDT, } 64 \%\end{array}$ & NR & $6.5 \%$ \\
\hline Kim, $2006^{10}$ & $\begin{array}{l}\text { Grade III: } \\
\text { PMT+CDT, } 84.2 \% \\
\text { CDT, 80.7\% } \\
\text { Grade II: } \\
\text { PMT+CDT, } 15.8 \% \\
\text { CDT, } 11.5 \% \\
\text { Grade I: } \\
\text { PMT+CDT, 0\% } \\
\text { CDT, } 7.7 \%\end{array}$ & NR & $\begin{array}{l}\text { PMT+CDT, } 15.4 \% \\
\text { CDT, } 25 \%\end{array}$ \\
\hline Kim, 2006 ${ }^{11}$ & $\begin{array}{l}\text { Grade III: } \\
\text { PMT+CDT, 82\% } \\
\text { CDT, 73\% } \\
\text { Grade II: } \\
\text { PMT+CDT, 19\% } \\
\text { CDT, 15\% } \\
\text { Grade I: } \\
\text { PMT+CDT, } 0 \% \\
\text { CDT, } 13 \%\end{array}$ & NR & NR \\
\hline Ozpak, $2016^{12}$ & 6 Months by Duplex, $85 \%$ & $\begin{array}{l}\text { VCSS at } 6 \text { months } \\
\text { Oedema, } 50 \% \\
\text { Skin pigmentation, 3\% } \\
\text { Pain, 3\% } \\
\text { Varicose veins, 3\% } \\
\text { Valvular incompetence, 24\% }\end{array}$ & NR \\
\hline Jia, $2016^{13}$ & 1 Year, $96.9 \%$ & Villalta scores: $<5,88 \% ; 5-9,5.9 \%$ & $10.9 \%$ \\
\hline Gagne, $2015^{14}$ & Duplex or CT venography, $94 \%$ & $\begin{array}{l}\text { VCSS at } 1 \text { year } \\
\text { Oedema, } 14 \% \\
\text { Skin changes, } 6 \% \\
\text { Pain, } 4 \% \\
\text { Varicose veins, } 2 \%\end{array}$ & NR \\
\hline Lee, $2013^{15}$ & $\begin{array}{l}1 \text { Year by Duplex or CT venography } \\
\text { AT+CDT, } 90.4 \% \\
\text { CDT, } 75.9 \%\end{array}$ & $\begin{array}{l}\text { AT+CDT, } 17.24 \% \\
\text { CDT, } 12.68 \%\end{array}$ & $\begin{array}{l}\text { AT+CDT, } 15.5 \% \\
\text { CDT, } 24 \%\end{array}$ \\
\hline Karahan, $2016^{16}$ & 6 Months by Duplex, $100 \%$ & Valvular incompetence, $12 \%$ & 0 \\
\hline Bozkurt, $2015^{17}$ & 6 Months by Duplex, $75 \%$ & NR & $7.1 \%$, due to irregular anticoagulant drug use \\
\hline Park, $2014^{18}$ & $\begin{array}{l}1 \text { Year by CT venography } \\
\text { MT, } 78 \% \\
\text { MT+CDT, } 80 \% \\
\text { CDT, } 71 \%\end{array}$ & No difference between MT/CDT & NR \\
\hline Shi, $2011^{19}$ & $\begin{array}{l}1 \text { Year by Duplex } \\
\text { Grade III, } 96 \% \\
\text { Grade II, } 4 \%\end{array}$ & $4 \%$ & NR \\
\hline Shi, $2009^{20}$ & $\begin{array}{l}13 \text { Months by Duplex } \\
\text { Grade III, } 50 \% \\
\text { Grade II, } 25 \%\end{array}$ & $6.2 \%$ & $0.97 \%$ \\
\hline Lee, $2006^{21}$ & 1 Year by CT venography, $85 \%$ & Valvular incompetence, $8 \%$ & NR \\
\hline
\end{tabular}

Abbreviations: AT = aspiration thrombectomy; CDT = catheter-directed thrombolysis; $C T$ = computed tomography; DVT = deep vein thrombosis; MT = mechanical thrombectomy; NR = not reported; PCDT = pharmacomechanical catheter-directed thrombolysis; PMT = percutaneous mechanical thrombectomy; RT = rheolytic thrombectomy; VCSS = Venous Clinical Severity Score 
TABLE 3. Complications of percutaneous mechanical thrombectomy $y^{6-21}$

\begin{tabular}{|c|c|c|c|}
\hline First author, year & Pulmonary embolism & Bleeding & Mortality \\
\hline Garcia, $2015^{6}$ & $0.3 \%$ & $\begin{array}{l}\text { Major, } 3.6 \% \text { (ICH, GIB secondary to gastritis or gastric cancer, } \\
\text { retroperitoneal bleeding, haemolytic anaemia and unspecified } \\
\text { bleeding requiring transfusion); minor, } 0.9 \%\end{array}$ & $0.3 \%$ (Unknown cause) \\
\hline Huang, $2015^{7}$ & No & 1 Minor epistaxis in CDT & No \\
\hline Arko, $2007^{8}$ & $\begin{array}{l}17 \%(5 / 30) \text { on } \mathrm{CT} \\
\text { (asymptomatic; all without } \\
\text { IVC filter) }\end{array}$ & No & No \\
\hline Lin, $2006^{9}$ & No & $\begin{array}{l}\text { PMT, } 4 \%, 0.2 \pm 0.3 \text { unit transfusion } \\
\text { CDT, } 6 \%, 1.2 \pm 0.7 \text { unit transfusion } \\
P<0.05\end{array}$ & No \\
\hline Kim, $2006^{10}$ & PMT, 5.3\%; CDT, 3.8\% & $\begin{array}{l}\text { PMT, 5.3\% (1 intra-abdominal haematoma required transfusion) } \\
\text { CDT, } 7.7 \% \text { (1 popliteal haematoma required surgical decompression) }\end{array}$ & No \\
\hline Kim, $2006^{11}$ & PMT, 4\%; CDT, 3\% & PMT, 4\%; CDT, 5\% & No \\
\hline Ozpak, $2016^{12}$ & NR & NR & NR \\
\hline Jia, $2016^{13}$ & No & No & No \\
\hline Gagne, $2015^{14}$ & No & No & No \\
\hline Lee, $2013^{15}$ & $\begin{array}{l}\text { CDT+AT, } 6 / 37 \\
\text { CDT, } 0 / 9\end{array}$ & 3 Haematoma in CDT & No \\
\hline Karahan, $2016^{16}$ & No & No & No \\
\hline Bozkurt, $2015^{17}$ & No & Minor subcutaneous bleeding at access site, $28.6 \%(4 / 14)$ & $\begin{array}{l}\text { Unrelated to } \\
\text { thromboemboli, } 1 / 14\end{array}$ \\
\hline Park, $2014^{18}$ & $2.2 \%$ & $\begin{array}{l}\text { Major } \\
\text { MT = } 0 \\
\text { MT+CDT = } 1 \mathrm{GIB} \\
\mathrm{CDT}=1 \mathrm{ICH} \\
\text { Minor } \\
\mathrm{MT}=2 \\
\mathrm{MT}+\mathrm{CDT}=3 \\
\mathrm{CDT}=6\end{array}$ & $2.2 \%$ in CDT \\
\hline Shi, $2011^{19}$ & No & Minor bleeding at access site, $8 \%(2 / 25)$ & No \\
\hline Shi, $2009^{20}$ & No & $\begin{array}{l}1 \text { Massive GIB } \\
\text { Subcutaneous bleeding, } 18.8 \%(3 / 16)\end{array}$ & $0.97 \%(1 / 103 \mathrm{ICH})$ \\
\hline Lee, $2006^{21}$ & No & No & No \\
\hline
\end{tabular}

Abbreviations: $\mathrm{AT}=$ aspiration thrombectomy; $\mathrm{CDT}=$ catheter-directed thrombolysis; $\mathrm{CT}=$ computed tomography; $\mathrm{GIB}=$ gastrointestinal bleeding; $\mathrm{ICH}=$ intracranial bleeding; IVC = inferior vena cava; MT = mechanical thrombectomy; NR = not reported; PMT = percutaneous mechanical thrombectomy

of hospital stay and $0.6 \pm 0.3$ days of ICU stay in the PMT group vs $8.4 \pm 2.3$ days of hospital stay and $2.4 \pm 1.2$ days of ICU stay in the CDT group; Pearson Chi squared, $\mathrm{P}<0.02$ to 0.04 ).

No statistically significant differences in venous patency or symptom improvement between the two groups were reported in this series of comparative studies.

\section{Comparison between types of thrombectomy devices}

Three studies compared outcomes of different thrombectomy devices. Garcia et $\mathrm{al}^{6}$ created the first prospective multi-centre (PEARL) registry to document the use of the AngioJet rheolytic device. A total of 329 patients were stratified into four treatment subgroups: (1) rheolytic thrombectomy (RT) alone; (2) RT plus CDT; (3) pharmacomechanical CDT
(PCDT), and (4) PCDT combined with CDT. Each of these subgroups differed in terms of the presence, timing, and delivery means of thrombolytic agents. Rheolytic thrombectomy was given before or after CDT in subgroup 2, and PCDT was defined as delivery of lytic agent through an AngioJet catheter. This registry demonstrated no statistical difference in venous patency rate between the subgroups, while a significant reduction in procedural time in non-CDT group was observed (Table 4). The investigators concluded that RT was effective and safe, and therefore, the needs for concomitant CDT and intensive care could potentially be reduced.

Shi et $\mathrm{al}^{20}$ and Arko et $\mathrm{al}^{8}$ compared the outcomes of Amplatz versus Rotarex and Trellis versus AngioJet devices, which again showed no significant differences in clinical outcomes between the two groups. 
TABLE 4. Secondary outcomes of percutaneous mechanical thrombectomy* 6,9-11,15,18

\begin{tabular}{|c|c|c|c|c|c|}
\hline First author, year & Subgroup & $\begin{array}{l}\text { Procedural time } \\
\text { (hours) }\end{array}$ & $\begin{array}{l}\text { Dosage of } \\
\text { thrombolytic (million } \\
\text { units) }\end{array}$ & Hospital stay (days) & Cost (US\$) \\
\hline Garcia, $2015^{6}$ & $\begin{array}{l}\text { RT } \\
\text { PCDT } \\
\text { PCDT+CDT } \\
\text { RT+CDT }\end{array}$ & $\begin{array}{l}1.4 \\
2 \\
22 \\
41 \\
P<0.0001\end{array}$ & & & \\
\hline \multirow[t]{2}{*}{ Lin, $2006^{9}$} & PMT & & & $\begin{array}{l}\text { Overall hospital stay: } \\
4.6 \pm 1.3 \\
\text { ICU stay: } \\
0.6 \pm 0.3\end{array}$ & $\begin{array}{l}\text { Total hospital cost: } \\
\$ 47742 \pm 19247 \\
\text { Hospital room cost: } \\
\$ 18392 \pm 8321\end{array}$ \\
\hline & CDT & & & $\begin{array}{l}\text { Overall hospital stay: } \\
8.4 \pm 2.3 \\
\text { ICU stay: } \\
2.4 \pm 1.2 \\
P<0.02 \text { to } 0.04\end{array}$ & $\begin{array}{l}\text { Total hospital cost: } \\
\$ 85301 \pm 24832 \\
\text { Hospital room cost: } \\
\$ 53632 \pm 1743 \\
P<0.01 \text { to } 0.02\end{array}$ \\
\hline Kim, $2006^{10}$ & $\begin{array}{l}\mathrm{PMT}+\mathrm{CDT} \\
\mathrm{CDT}\end{array}$ & $\begin{array}{l}30.3 \pm 17.8 \\
56.5 \pm 27.4 \\
P=0.001\end{array}$ & $\begin{array}{l}2.95 \pm 1.82 \\
6.70 \pm 5.9 \\
P=0.011\end{array}$ & & $\begin{array}{l}\$ 5128 \pm 2744 \\
\$ 10127 \pm 8836 \\
P=0.026\end{array}$ \\
\hline Kim, 2006 ${ }^{11}$ & $\begin{array}{l}\mathrm{PMT}+\mathrm{CDT} \\
\mathrm{CDT}\end{array}$ & $\begin{array}{l}26.3 \pm 16.6 \\
48.0 \pm 27.1 \\
P=0.0004\end{array}$ & $\begin{array}{l}2.7 \pm 1.8 \\
5.6 \pm 5.6 \\
P=0.008\end{array}$ & & \\
\hline Lee, $2013^{15}$ & $\begin{array}{l}\text { AT+CDT } \\
\text { CDT }\end{array}$ & $\begin{array}{l}22.0 \pm 7.1 \\
40.9 \pm 15.6 \\
P<0.001\end{array}$ & $\begin{array}{l}1.61 \pm 0.44 \\
22.01 \pm 7.10 \\
P<0.001\end{array}$ & & \\
\hline Park, $2014^{18}$ & $\begin{array}{l}\text { MT } \\
\text { MT+CDT } \\
\text { CDT }\end{array}$ & $\begin{array}{l}2.7 \pm 2 / 1 \\
18.2 \pm 8.2 \\
29.3 \pm 9.4 \\
P<0.001\end{array}$ & $\begin{array}{l}0 \\
5.13 \pm 3.72 \\
7.51 \pm 4.54 \\
P<0.001\end{array}$ & & \\
\hline
\end{tabular}

Abbreviations: AT = aspiration thrombectomy; CDT = catheter-directed thrombolysis; ICU = intensive care unit; $\mathrm{MT}=$ mechanical thrombectomy; PCDT = pharmacomechanical catheter-directed thrombolysis; PMT = percutaneous mechanical thrombectomy; RT = rheolytic thrombectomy

* Data are shown as mean \pm standard deviation, unless otherwise specified

\section{Use of adjunctive treatments}

Inferior vena cava filter placement, angioplasty, and stenting were the most commonly performed adjunctive treatments in addition to thrombectomy. Inferior vena cava filters were used in $46 \%$ to $100 \%$ of patients among 11 studies. The majority of the filters were removed shortly after the procedure without major complications. Lee et $\mathrm{al}^{15}$ reported that 6 of 37 patients had thrombus entrapment in prophylactic IVC filters in the thrombectomy group compared with 0 of 9 patients in the CDT alone group. Arko et $\mathrm{al}^{8}$ reported that $17 \%$ of patients showed asymptomatic pulmonary embolism on $\mathrm{CT}$ after thrombectomy, in which all patients did not receive IVC filters. This showed that prophylactic IVC filtration could be a useful measure for prevention of pulmonary embolism, especially in patients who undergo aggressive thrombectomy.

Angioplasty with or without stenting was performed in 15 studies, ranging from 14\% to $80 \%$ of patients. The two main indications were iliac vein compression syndrome (May-Thurner syndrome) and residual thrombus after thrombectomy. One study reported a significantly improved iliac vein patency rate in the group with stents $(28.95 \%)$ than without stents $(11.29 \%$; log rank test, $\mathrm{P}=0.026) .{ }^{15}$

\section{Discussion}

Catheter-directed thrombolysis and PMT are both emerging techniques for treatment of acute DVT of the lower extremities that have the advantage of early restoration of venous patency and thus reduction of post-thrombotic complications. A 2015 meta-analysis compared the efficacy of CDT plus anticoagulation versus that of anticoagulation alone in the treatment of proximal DVT. It showed that additional CDT was associated with significantly improved 6-month venous patency and PTS rates. However, there was a two-fold increase in bleeding complications in the CDT group, and concomitant close monitoring under intensive care setting has had a substantial economic burden. ${ }^{5}$ These two main reasons have precluded the incorporation of CDT into the standard treatment recommendation despite encouraging procedural outcomes.

As compared with CDT, PMT is another 
endovascular option that has provided promising clinical outcomes with better controlled bleeding risk. This review has served as a comprehensive overview of clinical and safety outcomes across different categories of thrombectomy devices. It demonstrated well that the procedural outcomes of both PMT alone and that with pharmacomechanical devices were non-inferior to that of CDT in treatment of acute DVT in the lower extremities. The rates of PTS, bleeding complications, and hospital costs of PMT were all shown to be favourable to those of CDT alone. In addition, the mortality risk of PMT was minimal and comparable to that of patients treated with anticoagulation alone: $0.4 \%$ recurrent fatal venous thromboembolism and $0.2 \%$ fatal major bleeding events. ${ }^{24}$ As illustrated in this review, the balanced risks and benefits of PMT provide a basis for the future initiation of randomised controlled trials on its use.

In addition, PMT is potentially superior to CDT especially in patients in whom thrombolysis therapy is contra-indicated. According to the Society of Interventional Radiology recommendations, CDT is absolutely contra-indicated in patients with recent cerebrovascular events, neurosurgery or intracranial trauma, active internal bleeding, and those with absolute contra-indications to anticoagulation. Other strong relative contra-indications are listed in the Standard of Practice ${ }^{25}$ : recent major surgery, obstetrical delivery or major trauma within 10 days, etc. These patients are prone to the development of DVT, and they have been conventionally treated with anticoagulation or IVC filters. Percutaneous mechanical thrombectomy is another option in this clinically challenging situation. Further studies on this particular group of high-risk patients are necessary to investigate the efficacy and safety of this novel technique.

Nevertheless, this review has several limitations. The studies were heterogeneous in terms of outcome measurements and the use of thrombectomy devices. Post-thrombotic syndrome was measured in terms of Villalta score, VCSS, or valvular incompetence rate. Although these systems were well-defined objective scales for monitoring and documentation of PTS, it was difficult to compare efficacy across studies. Similarly, the important index of venous patency rate was variously measured by Duplex ultrasound, CT venogram, or venography. Inaccuracies during direct comparison between studies were unavoidable.

Other adjunctive modalities in addition to the principal thrombectomy devices including iliac vein angioplasty, stenting, and prophylactic IVC filter were used in a major proportion of the studies. No standardised criteria were outlined for the usage of these devices, and they created a confounding factor during data analysis. With inadequate information on sub-categorisation of the study populations, analysis specific to each type of adjunctive devices was not feasible. Most of the studies were retrospective, and no randomised trials were available for quantitative analysis.

\section{Conclusion}

Percutaneous mechanical thrombectomy is a safe and effective treatment for acute iliofemoral DVT in terms of restoration of venous patency, prevention of DVT recurrence, PTS, and pulmonary embolism. The overall clinical outcomes of PMT are superior to those with anticoagulation alone. Compared with CDT alone, adjunctive PMT has a lower risk of PTS and bleeding complications. Randomised studies to demonstrate the efficacy of PMT versus anticoagulation and CDT and compare the efficacy of different types of PMT devices would be most beneficial to guide future strategies for treatment of acute proximal DVT.

\section{Author contributions}

All authors had full access to the data, contributed to the study, approved the final version for publication, and take responsibility for its accuracy and integrity.

Concept or design: All authors.

Acquisition of data: All authors.

Analysis or interpretation of data: PC Wong, YC Chan, Y Law.

Drafting of the manuscript: PC Wong, YC Chan, Y Law.

Critical revision: All authors.

\section{Conflicts of interest}

The authors declare no conflicts of interest.

\section{Declaration}

The paper was presented as an abstract in the 21st Asian Congress of Surgery by the Asian Surgical Association, 22-23 November 2017, Tokyo, Japan.

\section{Funding/support}

This research received no specific grant from any funding agency in the public, commercial, or not-for-profit sectors.

\section{References}

1. Kearon C, Kahn SR, Agnelli G, Goldhaber S, Raskob GE, Comerota AJ. Antithrombotic therapy for venous thromboembolic disease: American College of Chest Physicians Evidence-Based Clinical Practice Guidelines (8th edition). Chest 2008;133(6 Suppl):454S-545S.

2. Enden T, Haig Y, Kløw NE, et al. Long-term outcome after additional catheter-directed thrombolysis versus standard treatment for acute iliofemoral deep vein thrombosis (the CaVenT study): a randomised controlled trial. Lancet 2012;379:31-8.

3. Baldwin MJ, Moore HM, Rudarakanchana N, Gohel M, Davies AH. Post-thrombotic syndrome: a clinical review. J Thromb Haemost 2013;11:795-805.

4. Watson LI, Armon MP. Thrombolysis for acute 
deep vein thrombosis. Cochrane Database Syst Rev 2004;(4):CD002783.

5. $\mathrm{Du}$ GC, Zhang MC, Zhao JC. Catheter-directed thrombolysis plus anticoagulation versus anticoagulation alone in the treatment of proximal deep vein thrombosis-a meta-analysis. Vasa 2015;44:195-202.

6. Garcia MJ, Lookstein R, Malhotra R, et al. Endovascular management of deep vein thrombosis with rheolytic thrombectomy: final report of the prospective multicenter PEARL (Peripheral Use of AngioJet Rheolytic Thrombectomy with a Variety of Catheter Lengths) registry. J Vasc Interv Radiol 2015;26:777-85.

7. Huang CY, Hsu HL, Kuo TT, Lee CY, Hsu CP. Percutaneous pharmacomechanical thrombectomy offers lower risk of post-thrombotic syndrome than catheter-directed thrombolysis in patients with acute deep vein thrombosis of the lower limb. Ann Vasc Surg 2015;29:995-1002.

8. Arko FR, Davis CM 3rd, Murphy EH, et al. Aggressive percutaneous mechanical thrombectomy of deep venous thrombosis: early clinical results. Arch Surg 2007;142:5139

9. Lin PH, Zhou W, Dardik A, et al. Catheter-direct thrombolysis versus pharmacomechanical thrombectomy for treatment of symptomatic lower extremity deep venous thrombosis. Am J Surg 2006;192:782-8.

10. Kim HS, Patra A, Paxton BE, Khan J, Streiff MB. Catheterdirected thrombolysis with percutaneous rheolytic thrombectomy versus thrombolysis alone in upper and lower extremity deep vein thrombosis. Cardiovasc Intervent Radiol 2006;29:1003-7.

11. Kim HS, Patra A, Paxton BE, Khan J, Streiff MB. Adjunctive percutaneous mechanical thrombectomy for lowerextremity deep vein thrombosis: clinical and economic outcomes. J Vasc Interv Radiol 2006;17:1099-104.

12. Ozpak B, Ilhan G, Ozcem B, Kara H. Our short-term results with percutaneous mechanical thrombectomy for treatment of acute deep vein thrombosis. Thorac Cardiovasc Surg 2016;64:316-22.

13. Jia Z, Tu J, Zhao J, et al. Aspiration thrombectomy using a large-size catheter for acute lower extremity deep vein thrombosis. J Vasc Surg Venous Lymphat Disord 2016;4:167-71.

14. Gagne P, Khoury T, Zadeh BJ, Rajasinghe HA. A multicenter, retrospective study of the effectiveness of the trellis- 8 system in the treatment of proximal lower-extremity deep vein thrombosis. Ann Vasc Surg 2015;29:1633-41.
15. Lee JH, Kwun WH, Suh BY. The results of aspiration thrombectomy in the endovascular treatment for iliofemoral deep vein thrombosis. J Korean Surg Soc 2013;84:292-7.

16. Karahan O, Kutas HB, Gurbuz O, et al. Pharmacomechanical thrombolysis with a rotator thrombolysis device in iliofemoral deep venous thrombosis. Vascular 2016;24:4816.

17. Bozkurt A, Kırbaş İ, Kösehan D, Demirçelik B, Nazlı Y. Pharmacomechanical thrombectomy in the management of deep vein thrombosis using the cleaner device: an initial single-center experience. Ann Vasc Surg 2015;29:670-4.

18. Park KM, Moon IS, Kim JI, et al. Mechanical thrombectomy with Trerotola compared with catheter-directed thrombolysis for treatment of acute iliofemoral deep vein thrombosis. Ann Vasc Surg 2014;28:1853-61.

19. Shi HJ, Huang YH, Shen T, Xu Q. Percutaneous mechanical thrombectomy for acute massive lower extremity deep venous thrombosis. Surg Laparosc Endosc Percutan Tech 2011;21:50-3

20. Shi HJ, Huang $Y H$, Shen $T, X u$ Q. Percutaneous mechanical thrombectomy combined with catheterdirected thrombolysis in the treatment of symptomatic lower extremity deep venous thrombosis. Eur J Radiol 2009;71:350-5.

21. Lee KH, Han H, Lee KJ, et al. Mechanical thrombectomy of acute iliofemoral deep vein thrombosis with use of an Arrow-Trerotola percutaneous thrombectomy device. J Vasc Interv Radio 2006;17:487-95.

22. Porter JM, Moneta GL. Reporting standards in venous disease: an update. International Consensus Committee on Chronic Venous Disease. J Vasc Surg 1995;21:635-45.

23. Kahn SR, Partsch H, Vedantham S. Definition of postthrombotic syndrome of the leg for use in clinical investigations: a recommendation for standardization. J Thromb Haemost 2009;7:879-83.

24. Carrier M, Le Gal G, Wells PS, Rodger MA. Systematic review: case-fatality rates of recurrent venous thromboembolism and major bleeding events among patients treated for venous thromboembolism. Ann Intern Med 2010;152:578-89.

25. Vedantham S, Thorpe PE, Cardella JF, et al. Quality improvement guidelines for the treatment of lower extremity deep vein thrombosis with use of endovascular thrombus removal. J Vasc Interv Radiol 2009;20(7 Suppl):S227-39. 